Review Article

\title{
Nanoparticle Properties and Synthesis Effects on Surface-Enhanced Raman Scattering Enhancement Factor: An Introduction
}

\author{
Nathan D. Israelsen, Cynthia Hanson, and Elizabeth Vargis \\ Department of Biological Engineering, Utah State University, 4105 Old Main Hill, Logan, UT 84322, USA
}

Correspondence should be addressed to Elizabeth Vargis; vargis@usu.edu

Received 29 August 2014; Revised 1 November 2014; Accepted 2 November 2014

Academic Editor: Xiao-Feng Zhao

Copyright (c) 2015 Nathan D. Israelsen et al. This is an open access article distributed under the Creative Commons Attribution License, which permits unrestricted use, distribution, and reproduction in any medium, provided the original work is properly cited.

Raman spectroscopy has enabled researchers to map the specific chemical makeup of surfaces, solutions, and even cells. However, the inherent insensitivity of the technique makes it difficult to use and statistically complicated. When Raman active molecules are near gold or silver nanoparticles, the Raman intensity is significantly amplified. This phenomenon is referred to as surfaceenhanced Raman spectroscopy (SERS). The extent of SERS enhancement is due to a variety of factors such as nanoparticle size, shape, material, and configuration. The choice of Raman reporters and protective coatings will also influence SERS enhancement. This review provides an introduction to how these factors influence signal enhancement and how to optimize them during synthesis of SERS nanoparticles.

\section{Introduction}

Raman spectroscopy is a vibrational spectroscopy technique that has great promise for the identification of solids, liquids, and gases. Similar to infrared (IR) spectroscopy, Raman spectroscopy is a technique used to study molecular vibrations. One advantage that Raman spectroscopy has over traditional IR spectroscopy is that it avoids elaborate sample preparation. Despite this advantage, Raman spectroscopy was not initially as popular as IR spectroscopy due to difficulty in detecting Raman scattering. Statistically, for every 1-10 million photons bombarding a sample, only one will result in Raman scattering $[1,2]$. Thanks to technological advances such as mercury lamps, lasers, spectrometers, and charge-coupled devices (CCDs), Raman has gained popularity as a means of chemical identification [3]. Raman continued to gain traction with the aid of signal enhancement methods. In 1974, three scientists from Southampton University (Fleischmann, Hendra, and McQuillan) observed that the Raman spectrum of pyridine on a roughened silver electrode showed unusually large inelastic light scattering [4]. The phenomenon, later termed surface-enhanced Raman spectroscopy (SERS), can enhance the inelastic light scattering events by a factor of $10^{6}$ or more [5]. This enhancement allows researchers to develop extremely sensitive methods for quantification and identification of small molecules and molecular biomarkers.

Two mechanisms are behind the increased inelastic light scattering seen in SERS, electromagnetic and chemical enhancement. The electromagnetic enhancement is the dominant effect and is due to localized surface plasmon resonance (LSPR). LSPR is an optically excited electron wave resonance state on the surface of a nanostructure, which can increase both elastic (Rayleigh) and inelastic (Raman) light scattering from the sample [6]. The chemical enhancement mechanism is caused by molecular charge-transfer interactions between the molecule and the metallic surface $[7,8]$. Together, these mechanisms of enhancement increase the Raman intensity to a point where SERS can be used for applications which require greater molecular sensitivity.

There are two SERS methodologies, intrinsic and extrinsic SERS. Intrinsic SERS involves the direct measurement of the target molecule. In this process, a unique spectrum of the molecular vibration is acquired, which is referred to as a Raman signature. Intrinsic SERS is advantageous in understanding structural information about the target molecule and providing qualitative differences between similar samples. Intrinsic SERS has been used for many biological 


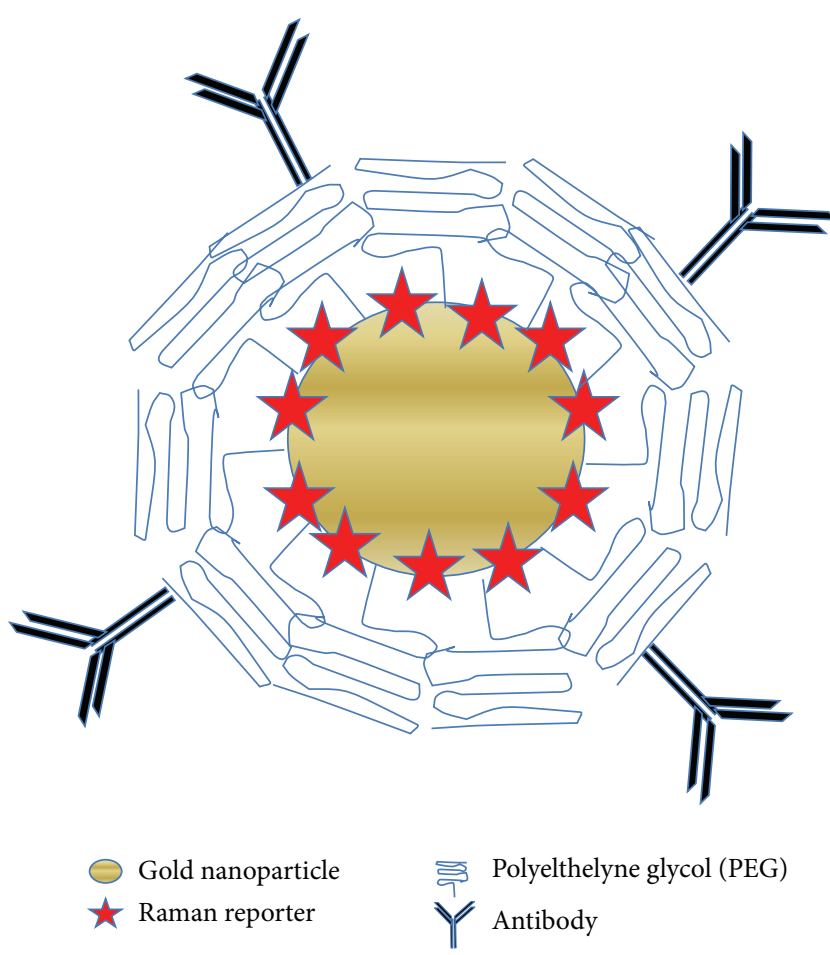

FIGURE 1: An illustration of an extrinsic SERS nanoparticle for targeting of a specific antigen.

applications such as detecting small molecules like glucose $[9,10]$, antioxidants [11], and amino acids [12]. It has also been used to detect macromolecules such as proteins [13] and DNA [14]. Drawbacks to intrinsic SERS include insensitivities of the molecule to Raman shifts and difficulty in detecting low concentrations. In cases of very dilute samples or where the sample is fairly Raman insensitive, extrinsic SERS provides an alternative mode of detection.

Extrinsic SERS is an indirect method of measuring target molecules because the acquired spectrum is of a Raman reporter rather than the target itself. A Raman reporter is a molecule that has inherently strong Raman active modes. An illustration of a nanoparticle used for extrinsic SERS is provided in Figure 1, where a Raman reporter molecule is bound to the metal surface and encased in a protective layer. This protective layer prevents nanoparticle aggregation and reporter leaching by steric hindrance and charge neutralization. The nanoparticle is then functionalized with an antibody or other ligand to target specific molecular sites. Nanoparticles for extrinsic SERS can be applied in a variety of situations where it is difficult to take intrinsic SERS measurements. For example, nanoparticles used for extrinsic SERS can target specific cell biomarkers [15] and cancer cells [16], while intrinsic SERS applications do not have targeting capabilities. In addition, nanoparticles for extrinsic SERS are synthesized to prevent aggregation in a variety of environments [17]. This trait accommodates in vivo measurements. For example, extrinsic SERS has been used to measure in vivo liver function [18]. In contrast, nanoparticles used in intrinsic SERS applications are susceptible to aggregation, preventing analysis in certain environments.
Regardless of the SERS method employed, the SERS enhancement factor (EF) is calculated in a variety of ways [19]. However, the simplest and most used definition relies on an estimate of the number of adsorbed reporter molecules and is calculated as follows:

$$
\mathrm{EF}=\frac{I_{\mathrm{SERS}} / N_{\mathrm{SERS}}}{I_{\text {Normal }} / N_{\text {Normal }}},
$$

where $I_{\text {SERS }}$ is the SERS intensity, $N_{\text {SERS }}$ is the number of molecules adsorbed to the metal surface in the SERS excitation volume, $I_{\text {Normal }}$ is the non-SERS intensity of the solution, and $N_{\text {Normal }}$ is the number of molecules in the non-SERS excitation volume. There has been some debate about this formula as it depends on several experimental conditions such as excitation wavelength, molecular species being evaluated, and molecular adsorption properties. Due to variations in these experimental conditions, it is difficult to make an accurate comparison of SERS EFs [19]. EFs are also difficult to compare as there are different types of EF measurements, the maximum EF and the average EF. The maximum EF, although larger, is seldom used in practice because it is difficult to locate SERS hot spots (locations of maximum SERS enhancement). The average SERS EF is the average enhancement for the scattering volume and is more repeatable. Maximum SERS EFs are typically on the order of $10^{10}$ while average SERS EFs are typically between $10^{4}$ and $10^{6}$ [19].

The lack of a rigorous definition of SERS EF and calculation errors has resulted in SERS EFs which have been reported as high as $10^{14}$ [5]. For example, in a study on single molecule detection of crystal violet, the SERS intensity of crystal violet is compared to the non-SERS intensity of methanol. This comparison resulted in a high SERS EF which is inaccurate because the author failed to account for the non-SERS intensity of crystal violet [19]. Other sources of error include failing to account for the following parameters: molecule orientation and surface selection rules [20], differentiation between average and maximum EF [21], and photobleaching [22]. In addition, errors can result from inaccurate measurements of metal bound analyte concentration due to shape irregularity and surface roughness [23]. A detailed discussion and rigorous definition of EF has been presented by Le Ru et al., and further details will not be discussed here [19].

The SERS enhancement effect has been observed with alkaline metals [24], various transition and noble metals $[24,25]$, metals oxides [25] and even semiconductor materials like silicone and graphene $[25,26]$. The SERS enhancement of semiconductors is primarily due to chemical mechanisms and is weaker than the enhancement observed with noble metals [25]. Despite the lower enhancement, semiconductors have potential applications in the development of photodetectors and biosensors [27].

Typically, SERS is observed with coinage metals such as gold, silver, and copper due to the large SERS enhancement they produce. Of these metals, silver provides the highest enhancement factor due to its absorption properties. Optical absorption in metals is increased by interband transitions. The interband transition of silver is found in the ultraviolet range. As a result, there will be less absorption in the visible 
or near-IR Raman wavelengths resulting in large SERS intensities. In contrast, gold and copper have interband transitions in the visible wavelength range which results in a decrease in the maximum SERS intensity $[28,29]$. Despite the greater enhancement capability of silver, gold is often used as it is more stable, is biocompatible, and has an easier surface chemistry than silver [30]. The decision to use gold or silver will depend on several factors such as application, excitation wavelength, LSPR wavelength, and experimental setup.

It has been reported that SERS enhancement is dependent on the distance between the SERS surface and the molecule of interest [31]. Although the target molecule does not need to be touching the surface for SERS enhancement to occur, literature suggests that it needs to be within 1-30 $\mathrm{nm}$ for reasonable SERS enhancement [32-34]. The actual distance for SERS enhancement is highly dependent on the nanoparticle surface. Calculations for metallic spherical nanoparticles have predicted that the SERS intensity will decay with increasing distance, $r$, between the target molecule and particle surface. The distance for electromagnetic enhancement is proportional to $r^{-12}$. Due to the addition of surface molecules, the decay relationship increases slightly and is proportional to $r^{-10}[31,33]$.

To effectively optimize nanoparticle EF, the LSPR peak should be taken into account. Initially, it was proposed that the largest amount of Raman scattering should be observed when the particle was excited at its LSPR peak [35]. Experimental studies have found that the optimal excitation wavelength will be slightly blue-shifted from the nanoparticle LSPR peak [36]. One approach to estimating the optimal excitation wavelength for SERS enhancement is to design nanoparticles with an LSPR peak $\left(\lambda_{\text {LSPR }}\right)$ between the excitation wavelength $\left(\lambda_{0}\right)$ and the Raman wavelength of the interest $\left(\lambda_{R}\right)$. This can be expressed as $\lambda_{\text {LSPR }}=$ $\left(\lambda_{0}+\lambda_{R}\right) / 2$ [37]. This approach has been validated by a number of independent researchers and appears to apply to many nanostructures investigated [37-39]. However, there are deviations from this rule due to the excitation wavelength used as well as the shape of the nanoparticle [40]. As a general guideline, SERS enhancement has been observed for excitation wavelengths from $600 \mathrm{~nm}$ to $1200 \mathrm{~nm}$ for gold and from $400 \mathrm{~nm}$ to $1000 \mathrm{~nm}$ for silver [41].

To synthesize SERS nanoparticles with an optimal EF for intrinsic applications, it is requisite to understand how a nanoparticle's material, size, shape, and configuration influence EFs. Synthesis of nanoparticles for extrinsic SERS applications requires additional understanding of how Raman reporters and protective layers influence EF. This paper provides an introduction to how these factors influence EF (Sections 2 and 3 ) and how to control them during synthesis (Sections 4 and 5).

\section{Enhancement Dependency on Nanoparticle Properties for Intrinsic SERS}

2.1. Enhancement Dependency on Nanoparticle Size. Enhancement and LSPR dependency on size is demonstrated in Figure 2, which shows the theoretical (Mie theory) extinction

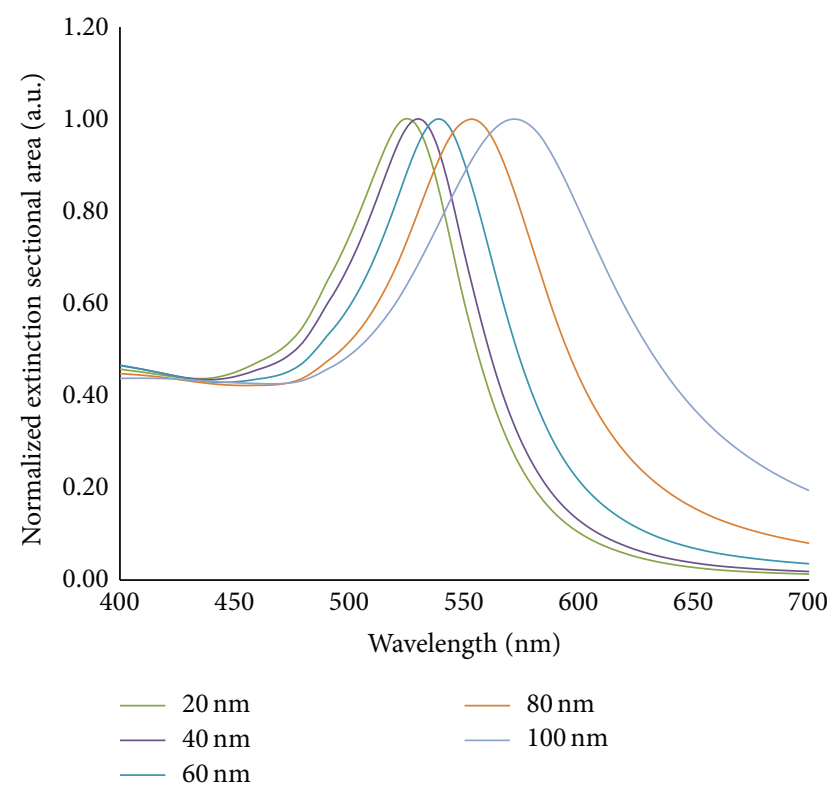

FIGURE 2: Normalized extinction cross-sectional area for spherical gold nanoparticles of diameters ranging from $20-100 \mathrm{~nm}$. Data was obtained from a Mie theory simulation available online [42].

cross section for gold spheres ranging from 20 to $100 \mathrm{nms}$ in diameter. The Mie theory is the solution to the Maxwell equations for how light interacts with a spherical particle. The theory predicts that the smallest spheres have a maximum LSPR at shorter wavelengths. Details concerning the Mie theory can be found in literature and will not be covered here $[43,44]$. The relationship between LSPR wavelength and particle size has also been demonstrated experimentally [4547].

The effect of nanoparticle size on SERS enhancement has been studied by several researchers [48-52]. Previous studies show that spherical gold particles with a $50 \mathrm{~nm}$ diameter produced the maximum SERS EF [48, 51]. Others have reported the optimal size particles for SERS enhancement to be in a range from 30 to $100 \mathrm{~nm}[50,52]$. Regardless of the exact size for optimal enhancement, there is an effective SERS range. When particles are too small, the effective conductivity and light scattering properties, which are needed for SERS enhancement, diminish [50]. As particles increase in size, the SERS effect increases as it depends on the number of electrons available [53]. When the particle size approaches the scale of the excitation wavelength, the particles become preferentially excited in nonradiative modes, leading to a diminished SERS effect [50].

2.2. Enhancement Dependency on Shape. Variations in particle shape can expand the LSPR range. For example, gold nanorods with aspect ratios from 1 to 19 have a LSPR peak range from $508 \mathrm{~nm}$ to $2135 \mathrm{~nm}$ [54]. Controlling particle shape and size allows researchers to accurately tune nanoparticles for a specific LSPR peak and optimize EF. Shape also influences SERS enhancement due to locations of high curvature such as sharp corners or tips. These locations 
produce unusually large electromagnetic enhancement. This enhancement is referred to as the lightning rod effect because similar to a pointed lightning rod, the electric field induced at the tip will be much stronger than other areas on the surface [55]. The influence of the lightning rod effect can be observed near high curvature points on many different shapes [56, 57]. The lighting rod effect allows nanostructures to act as an optical antenna providing an enhanced electromagnetic field [58-60]. More details about the lighting rod effect can be found in literature $[61,62]$.

\subsection{Enhancement Dependency on Material and Configuration.} SERS core-shell configurations can be made by coating a nanoparticle with gold or silver. Some common core materials include organic polymers [63-65], silica [66-68], iron oxide $\left(\mathrm{Fe}_{3} \mathrm{O}_{4}\right)[69,70]$, or other metals [71-73]. In core shell configurations, the materials used [73], the overall particle size [74], the shell thickness [75], and the core/shell ratio [74] will influence SERS EF due to shifts in the LSPR peak. For the purpose of this paper, only the influence of core-shell materials and shell thickness on SERS enhancement factor will be covered.

When two metals with different dielectric constants are placed next to each other in a core shell configuration, a LSPR shift will occur. By using the Mie theory, researchers have predicted that increasing the shell thickness of a nanoparticle will shift the LSPR peak to shorter wavelengths $[75,76]$. This shift has been confirmed experimentally in a number of cases [75-77]. For example, Oldenburg et al. [75] observed a blue shift in the extinction peak as the gold shell thickness increased over a silica core once the shell was fully formed. The same trend can be seen in nanorods with a core-shell configuration. Ma et al. [76] observed how the LSPR peak shifted to lower wavelengths as the shell thickness increased for silver coated gold nanorods. Not only was there a blue shift, but the intensity also increased according to shell thickness. In the case of silver-gold core-shell arrangements, there will be a red shift as gold has a plasmon resonance peak at a longer wavelength than silver. As the gold shell thickness increases, the absorbance peak also diminishes [78, 79]. In addition to these changes, silver-gold or gold-silver coreshell arrangements create two plasmon resonance peaks [76, $78,80]$ corresponding to silver and gold. Changes in LSPR wavelengths can be modified using core-shell nanoparticles by controlling composition materials and shell thickness among other parameters. The LSPR can be tuned to a position to create a maximum enhancement factor by proper placement in relationship to the excitation and emission wavelengths [40].

Another approach to SERS enhancement is due to SERS hot spots. They are locations between particle aggregates which amplify the electromagnetic field near the particle. Researchers have often used nanoparticle junctions or aggregates to create repeatable hot spot locations for single molecule SERS measurements [81-83]. By carefully controlling the placement of the reporter molecule within the particle junction, a maximum SERS EF is observed because hot spot locations are known in advance [84].
TABLE 1: A list of molecules used for SERRS enhancement for excitation wavelengths $\left(\lambda_{0}\right)$ from $514-785 \mathrm{~nm}$ and their associated absorption maximums $\left(\lambda_{\max }\right)$.

\begin{tabular}{|c|c|c|c|}
\hline Raman reporter & $\lambda_{\max }(\mathrm{nm})$ & $\lambda_{0}(\mathrm{~nm})$ & Reference \\
\hline Carboxyfluorescein (FAM) & 494 & 514.5 & {$[85]$} \\
\hline Rhodamine $6 \mathrm{G}$ (R6G) & 524 & $514.5,532$ & {$[85-88]$} \\
\hline TRITC-DHPE & 540 & 514.5 & [89] \\
\hline $\begin{array}{l}\text { Carboxy-X-rhodamine } \\
\text { (ROX) }\end{array}$ & 585 & $514.5,632.8$ & {$[85]$} \\
\hline BIODIPY TR-X & 588 & 632.8 & [85] \\
\hline Crystal violet (CV) & 590 & $514.5,647.1$ & {$[86,90]$} \\
\hline Malachite green (MG) & 618 & 632.8 & [91] \\
\hline $\begin{array}{l}\text { Malachite green } \\
\text { isothiocyanate (MGITC) }\end{array}$ & 625 & 632.8 & {$[91]$} \\
\hline Methylene blue & $570-760^{\dagger}$ & 785 & {$[92,93]$} \\
\hline Cy5.5 & 683 & 632.8 & {$[85]$} \\
\hline $\begin{array}{l}3,3^{\prime}- \\
\text { diethylthiatricarbocyanine } \\
\text { iodide (DTTC) }\end{array}$ & 765 & 785 & {$[94]$} \\
\hline \multicolumn{4}{|c|}{$\begin{array}{l}{ }^{+} \text {Methylene blue }(\mathrm{MB}) \text { has various different maximum absorption points } \\
\text { due to the formation of molecular aggregates and protonation states. The } \\
\text { formation of dimer and trimer aggregates and MB protonation causes a shift } \\
\text { in the maximum absorption. As a result, the maximum absorption is directly } \\
\text { related to the state of the MB molecule during measurement [93]. }\end{array}$} \\
\hline
\end{tabular}

\section{Enhancement Dependency on Nanoparticle Properties for Extrinsic SERS}

3.1. Enhancement Dependency on Raman Reporter Properties. Fluorescent dyes and other chromophores are often used as Raman reporters because they have large Raman scattering cross sections, are photostable, and have resonance Raman capabilities. In resonance Raman scattering, the laser excitation wavelength is matched to the reporter molecule's absorption maximum and an approximate $10^{3}-10^{6}$ increase in Raman scattering occurs [95]. By carefully matching the excitation wavelength and nanoparticle composition, surface-enhanced resonance Raman scattering (SERRS) can be observed. SERRS is especially suited for extrinsic measurements if the reporter molecule and excitation wavelengths are known before analysis and can be selected to provide the highest enhancement possible. Table 1 shows molecules that have been used for SERRS studies.

Other characteristics which are important when selecting a Raman reporter molecule are its polarizability, photostability, and binding affinity. Polarizability is the ability of an external electromagnetic field to produce a change in the electron distribution in the molecule. Polarizable molecules have bond configurations which allow an excitation of the vibrational modes of the molecule in response to incident light. A Raman active molecule has a net change in its polarizability when excited by light which causes a shift in the wavelength of the scattered light [96]. Raman reporters should be highly Raman active.

The photostability of a Raman reporter molecule plays an important role in the SERS EF and in determining 
the maximum laser power to use. Photobleaching effects can account for SERS EF differences on the order of $10^{2}-10^{3}$, which is comparable to the EF contribution due to chemical enhancement [97]. Photobleaching effects are related not only to the power of the incident light but also to the actual electromagnetic enhancement experienced by the reporter. As a result, even non-resonant molecules may experience photobleaching [98]. Due to the photobleaching effect, SERS nanoparticles experience a laser power dependent SERS EF. Lower laser power will reduce photobleaching effects and result in more consistent SERS measurements [97]. The use of near IR or IR lasers for excitation will also reduce the photobleaching effect and is recommended for photosensitive reporters [99].

Molecules with strong binding affinities to a gold or silver surface give stronger enhancement due to the chemical enhancement mechanism [91]. High binding affinity molecules that are frequently used are thiol or amine containing molecules because they will easily bind to the gold surface through the gold thiolate bond. Also, positively charged molecules such as crystal violet can associate with the negatively charged nanoparticle surface and are frequently used as Raman reporters [90]. Qian et al. showed how strong binding affinity affects the SERS enhancement factor when using two very similar Raman reporters: malachite green and malachite green isothiocyanate (MGITC) [91]. Although both molecules have similar Raman scattering cross sections and vibrational modes, MGITC showed an approximate 200fold higher SERS EF under identical measurement conditions. They attributed this EF increase to stable anchoring of the MGITC molecule (by the isothiocyanate group) which enabled charge transfer and chemical enhancement to occur [91].

3.2. Enhancement Dependency on Protective Coating. The SERS protective coating can influence the enhancement effect due to its laser transparency and binding affinity. Coating materials must allow laser light to penetrate the coating and interact with the Raman reporters. Many materials have been used and include polymers [100], silica [101], or proteins [102]. Some coating materials can have an adverse effect on SERS response. For example, Huang et al. [103] observed a 60\% decrease in the SERS signal due to the silica coating. Often, (3-mercaptopropyl)trimethoxysilane (MPTMS) is used as a silane functionalizing group because of its ability to bind to the gold surface through a thiolate bond. When the reporter and MPTMS are added simultaneously, the sulfur group binds preferentially, decreasing reporter density and the resulting SERS intensity [104]. This effect highlights the importance of controlling the reporter density on the particle surface as this will have an impact on the SERS enhancement.

\section{Controlling Nanoparticle Geometry}

The two main approaches for nanoparticle synthesis are the top-down and bottom-up approaches. The top-down approach starts with larger particles and disperses them to smaller particles. The bottom-up approach starts with smaller particles which build upon each other to form nanoparticles [105]. Top-down approaches typically produce particles in solution that are not as stable or reproducible as those produced from bottom-up approaches [105]. Therefore, this section will focus on bottom-up methods. Some examples of bottom-up approaches include chemical reduction [106109], sonochemical synthesis [63, 110, 111], photochemical reduction [112], and radiolytic reduction [113]. As this is an introduction to optimal SERS nanoparticle synthesis, the most common chemical reduction methods will be covered.

Chemical reduction methods for synthesis of gold or silver nanoparticles incorporate the use of capping and reducing agents. A capping agent binds to the surface of the nanoparticle and prevents aggregation by repulsive or steric forces. It can also be used to functionalize nanoparticles in extrinsic SERS applications by providing a link to bind the nanoparticle to reporters and antibodies. Some examples include sodium citrate [106], dodecanethiol [107], thiol polyethylene-glycol (PEG) [114], cetrimonium bromide (CTAB) [115], tannic acid [116], hydroxylamine hydrochloride [117], and polyvinylpyrrolidone (PVP) [118]. A reducing agent changes a metal ion in solution to a solid particle. For example, sodium borohydride $\left(\mathrm{NaBH}_{4}\right)$ reduces $\mathrm{Au}^{+3}$ to solid $\mathrm{Au}$ in the Brust-Schiffrin method of gold nanoparticle synthesis [107]. Some common reducing agents used for gold nanoparticle synthesis include sodium citrate [106], $\mathrm{NaBH}_{4}$ [107], and hydroxylamine [69].

A common chemical reduction method is the Turkevich method. It was introduced in the early 1950s [106] and was a modification of a previous method to synthesize gold nanoparticles by Hauser and Lynn [119]. Although the Turkevich method was introduced over 60 years ago, it is one of the most commonly used methods today and elements of this method can be seen in many techniques to coat particles and surfaces with gold or silver. In this method, sodium citrate is added to a boiling aqueous solution of chloroauric acid $\left(\mathrm{HAuCl}_{4}\right)$. Sodium citrate acts as the capping and reducing agent. The Lee-Meisel method [108] uses the same concept to form silver nanoparticles by reducing silver using sodium citrate. The Turkevich and Lee-Meisel methods produce irregularly shaped nanoparticles $[120,121]$ with diameters ranging from 10 to $150 \mathrm{~nm}$ [122] and 60 to $200 \mathrm{~nm}$ [120], respectively. It should be noted that several adaptations have been incorporated to these methods to improve control of nanoparticle size and shape $[120,123]$.

Nanoparticle size is most affected by the strength and concentration of the reducing agent. Stronger reducing agents produce smaller nanoparticles and weaker reducing agents producing larger particles [124]. Sodium citrate is a weak reducing agent, and as such, the Turkevich and Lee-Meisel methods produce relatively large nanoparticles. The BrustSchiffrin method [107] uses sodium borohydride as a reducing agent, which is much stronger. As a result, this method creates well-dispersed gold nanoparticles ranging from 1 to $3 \mathrm{~nm}$ [107].

The size distribution of particles can be refined by controlling the nucleation and growth stages during chemical reduction. In the nucleation process, metal atoms combine to 
form clusters and finally crystal nuclei. In the growth step, the crystal nuclei or "seeds" grow in size to form nanoparticles. These steps can be separated for greater control of size and shape and the process is referred to as seed-mediated growth. In these methods, direct synthesis is used to produce the seeds. Consistent seeds are vital to control subsequent nanoparticle shape and size $[115,125,126]$. Several journal articles give further details of seeding methods [115, 120, 125129] and will not be covered here.

Nanoparticle shape can be controlled by adding surfactants during synthesis which will cause a change in surface energy and control particle aggregation. The surfactant will stabilize specific crystal planes in the growing nanostructure, allowing controlled growth on that plane [130]. By carefully choosing a surfactant and particle material, multiple nanoparticle shapes have been created including nanorods $[111,131]$, nanocubes [132], nanostars [92, 133], nanotriangles $[36,134]$, nanoplates [135], nanowires [136], and nanoshells [137-139].

\section{Tailoring Nanoparticles for Extrinsic SERS Applications}

5.1. Coating Extrinsic Nanoparticles. A common approach for coating Raman reporter encoded gold nanoparticles is to bind a thiol PEG molecule to the gold nanoparticle surface by a gold thiolate bond. For citrate stabilized gold nanoparticles, the thiol group will easily replace the citrate molecule and will provide stabilization [140]. The surface charge of the particles can be controlled by varying the molecular weight of PEG. A simple one-step method to synthesize PEG coated gold nanoparticles has recently been developed [141]. Briefly, it involves heating an aqueous $\mathrm{PEG}$ and $\mathrm{NaOH}$ solution at $50^{\circ} \mathrm{C}$. An aqueous solution of $\mathrm{HAuCl}_{4}$ is then added rapidly while slowly increasing the temperature to $80^{\circ} \mathrm{C}$. This method avoids the two-step process of making the gold nanoparticles and then coating with PEG. It also avoids the use of additional chemicals as reduction agents.

The use of a silica shell for coating Raman reporter encoded nanoparticles provides increased stability even in the presence of multiple solvents [142]. To coat the Raman reporter labeled nanoparticles, aminosilanes, such as (3-aminopropyl)triethoxysilane (APTES) or (3-aminopropyl)trimethoxysilane (APTMS), are used to functionalize the particle surface. Tetraethyl orthosilicate (TEOS) or another activated cross-linking agent is used to form the silca shell. TEOS is highly sensitive to water and forms silica dioxide when added to the aminosilane activated gold nanoparticles [143]. Upon formation of the silica shell, these particles are extremely stable and have been used for in vivo SERS measurements without loss of the SERS response [16].

5.2. Addition of Antibodies or Other Affinity Ligands. An important factor in the design of extrinsic SERS nanoparticles' is the ability for targeting of specific recognition sites through antibody antigen interactions [15] or DNA hybridization [143]. To bind these ligands to the particle shell, multiple techniques have been used [138, 143-145]. A common approach is the use of a PEG N-hydroxysuccinimide (NHS) ester for covalent linkage to primary amines in the $\mathrm{N}$ terminus or lysine residues. For additional information see [146]. Ligands can be bound to silica coated particles using (3-glycidoxypropyl)methyldiethoxysilane (GPTMS). Li et al. [143] used GPTMS to provide a link between a silica surface and DNA. This binding is due to a highly reactive epoxide group associated with GPTMS. Another approach of surface functionalization involves the addition of a carboxyl group to the surface of the silica shell for further bioconjugation through EDC carbodiimide and NHS chemistry. In this approach, the nanoparticles are first suspended in dimethylformamide (DMF) and then 3(triethoxysilylpropylcarbamoyl)butyric acid is added to the nanoparticles' silica surface. The resulting $\mathrm{COOH}$ functionalized silica particles can be used for bioconjugation of oligonucleotides or antibodies [147].

\section{Future Applications}

6.1. Hybrid Intrinsic/Extrinsic SERS. A potential application of SERS nanoparticles is the development of hybrid nanoparticles. Extrinsic methods generally use Raman reporters and antibodies for indirect sensing of the target molecule, while intrinsic methods directly enhance the target molecules' Raman spectrum. Hybrid applications combine elements of both intrinsic and extrinsic methods. This results in a nanoparticle which can target and enhance the Raman signature of a molecule providing structural information. An approach to develop hybrid SERS nanoparticles is to expose parts of the nanoparticle surface so that it can interact with the target molecule. Kneipp et al. [148] used unprotected nanoparticles with bound reporter molecules to investigate cellular uptake and endosome formation inside various different cells [148-150]. The presence of the reporter molecule enabled multiplexing and specific particle localization. Additionally, the unprotected particle surface allowed the detection of Raman bands related to intracellular components such as lipids, nucleotides, and proteins [149].

A critical factor in the design of hybrid nanoparticles is minimizing the distance between the particle and the target molecule. As mentioned previously, the target molecule must be within approximately $1-30 \mathrm{~nm}$ for SERS enhancement to occur. Several researchers have applied different techniques to minimize the distance between the nanoparticle and the target using hybrid SERS applications. For example, Hodges et al., used antibody conjugated gold nanoparticles to bind to specific cell surface regions for targeted SERS enhancement [151]. The large size of the antibody resulted in a large separation between the nanoparticle and the target molecule which limited the SERS enhancement. In order to increase the SERS enhancement, the distance between the metal surface and the target molecule was reduced by using the gold nanoparticles as nucleation centers for silver deposition. The deposited silver allowed for SERS enhancement of only the region around the antibody binding site [151]. Another technique to minimize the distance between the nanoparticle and the target molecule is through the use of antibody 
fragments. An antibody fragment is composed of the antigen binding site but does not contain the tail region. As a result the binding capacity is preserved but the total distance occupied by the fragment is less than that of a full antibody. Wang et al. demonstrated that nanoparticles with conjugated antibody fragments can significantly increase the SERS intensity while still maintaining targeting capabilities [152].

\section{Summary}

The optimization of SERS nanoparticles to achieve the highest enhancement factor is a complex process that incorporates multiple variables which must be precisely controlled by synthesis methods. Nanoparticles for intrinsic SERS applications can be synthesized to control composition, size, shape, and configuration which will affect the LSPR peak location and SERS EF. Smaller particles result in a shorter LSPR wavelength in accordance with the Mie theory. Size can be set during chemical synthesis by proper control of nucleation and growth steps as well as the strength of the reducing agent. Stronger reducing agents will result in smaller particles. Shape will also influence SERS enhancement due to electromagnetic enhancement at edges or sharp corners. Shape can be controlled during synthesis by the addition of surfactants. The proper choice of Raman reporters and protective coatings will determine the enhancement associated with extrinsic SERS. An appropriate Raman reporter will have a strong binding affinity to the nanoparticle surface and allow for resonance Raman enhancement. Care must be taken in the synthesis of the protective layer to prevent loss of the SERS signal. Controlled synthesis of intrinsic and extrinsic SERS nanoparticles can be tuned for specific systems and applications providing optimal SERS enhancement.

\section{Conflict of Interests}

The authors declare that there is no conflict of interests regarding the publication of this paper.

\section{Authors' Contribution}

Nathan D. Israelsen and Cynthia Hanson contributed equally to this work.

\section{References}

[1] C. L. Zavaleta, M. F. Kircher, and S. S. Gambhir, "Raman's "effect" on molecular imaging," Journal of Nuclear Medicine, vol. 52, no. 12, pp. 1839-1844, 2011.

[2] E. Le Ru and P. G. Etchegoin, "Basic principles," in Principles of Surface-Enhanced Raman Spectroscopy: And Related Plasmonic Effects, pp. 7-25, Elsevier Science, Boston, Mass, USA.

[3] W. H. Nelson and J. F. Sperry, "UV resonance Raman spectroscopic detection and identification of bacteria and other microorganisms," in Modern Techniques for Rapid Microbiological Analysis, pp. 97-143, VCH, New York, NY, USA, 1991.

[4] M. Fleischmann, P. J. Hendra, and A. J. McQuillan, "Raman spectra of pyridine adsorbed at a silver electrode," Chemical Physics Letters, vol. 26, no. 2, pp. 163-166, 1974.
[5] K. Kneipp, Y. Wang, H. Kneipp et al., "Single molecule detection using surface-enhanced Raman scattering (SERS)," Physical Review Letters, vol. 78, no. 9, pp. 1667-1670, 1997.

[6] P. Alonso-González, P. Albella, M. Schnell et al., "Resolving the electromagnetic mechanism of surface-enhanced light scattering at single hot spots," Nature Communications, vol. 3, article 684, 2012.

[7] L. Jensen, C. M. Aikens, and G. C. Schatz, "Electronic structure methods for studying surface-enhanced Raman scattering," Chemical Society Reviews, vol. 37, no. 5, pp. 1061-1073, 2008.

[8] A. Campion, J. E. Ivanecky, C. M. Child, and M. Foster, "On the mechanism of chemical enhancement in surface-enhanced raman scattering," Journal of the American Chemical Society, vol. 117, no. 47, pp. 11807-11808, 1995.

[9] O. Lyandres, J. M. Yuen, N. C. Shah, R. P. VanDuyne, J. T. Walsh Jr., and M. R. Glucksberg, "Progress toward an in vivo surface-enhanced Raman spectroscopy glucose sensor," Diabetes Technology and Therapeutics, vol. 10, no. 4, pp. 257-265, 2008.

[10] C. R. Yonzon, C. L. Haynes, X. Zhang, J. T. Walsh Jr., and R. P. Van Duyne, "A glucose biosensor based on surface-enhanced Raman scattering: improved partition layer, temporal stability, reversibility, and resistance to serum protein interference," Analytical Chemistry, vol. 76, no. 1, pp. 78-85, 2004.

[11] M. Dendisova-Vyskovska, G. Broncova, M. Clupek, V. Prokopec, and P. Matejka, "In situ SERS spectroelectrochemical analysis of antioxidants deposited on copper substrates: what is the effect of applied potential on sorption behavior?" Spectrochimica Acta-Part A: Molecular and Biomolecular Spectroscopy, vol. 99, pp. 196-204, 2012.

[12] E. Podstawka, Y. Ozaki, and L. M. Proniewicz, "Part III: surface-enhanced raman scattering of amino acids and their homodipeptide monolayers deposited onto colloidal gold surface," Applied Spectroscopy, vol. 59, no. 12, pp. 1516-1526, 2005.

[13] L.-J. Xu, C. Zong, X.-S. Zheng, P. Hu, J.-M. Feng, and B. Ren, "Label-free detection of native proteins by surface-enhanced Raman spectroscopy using iodide-modified nanoparticles," Analytical Chemistry, vol. 86, no. 4, pp. 2238-2245, 2014.

[14] A. Barhoumi, D. Zhang, F. Tam, and N. J. Halas, "Surfaceenhanced raman spectroscopy of DNA," Journal of the American Chemical Society, vol. 130, no. 16, pp. 5523-5529, 2008.

[15] C. T. Nguyen, J. T. Nguyen, S. Rutledge, J. Zhang, C. Wang, and G. C. Walker, "Detection of chronic lymphocytic leukemia cell surface markers using surface enhanced Raman scattering gold nanoparticles," Cancer Letters, vol. 292, no. 1, pp. 91-97, 2010.

[16] X. Qian, X.-H. Peng, D. O. Ansari et al., "In vivo tumor targeting and spectroscopic detection with surface-enhanced Raman nanoparticle tags," Nature Biotechnology, vol. 26, no. 1, pp. 83-90, 2008.

[17] J. Manson, D. Kumar, B. J. Meenan, and D. Dixon, "Polyethylene glycol functionalized gold nanoparticles: the influence of capping density on stability in various media," Gold Bulletin, vol. 44, no. 2, pp. 99-105, 2011.

[18] C. L. Zavaleta, B. R. Smith, I. Walton et al., "Multiplexed imaging of surface enhanced Raman scattering nanotags in living mice using noninvasive Raman spectroscopy," Proceedings of the National Academy of Sciences of the United States of America, vol. 106, no. 32, pp. 13511-13516, 2009.

[19] E. C. Le Ru, E. Blackie, M. Meyer, and P. G. Etchegoint, "Surface enhanced raman scattering enhancement factors: a comprehensive study," The Journal of Physical Chemistry C, vol. 111, no. 37, pp. 13794-13803, 2007. 
[20] D. V. Chulhai and L. Jensen, "Determining molecular orientation with surface-enhanced raman scattering using inhomogenous electric fields," Journal of Physical Chemistry C, vol. 117, no. 38, pp. 19622-19631, 2013.

[21] C. J. Choi, Z. Xu, H.-Y. Wu, G. L. Liu, and B. T. Cunningham, "Surface-enhanced Raman nanodomes," Nanotechnology, vol. 21, no. 41, Article ID 415301, 2010.

[22] P. G. Etchegoin, E. C. Le Ru, R. C. Maher, and L. F. Cohen, "Enhancement factor averaging and the photostability of probes in SERS vibrational pumping," Physical Chemistry Chemical Physics, vol. 9, no. 35, pp. 4923-4929, 2007.

[23] P. H. C. Camargo, L. Au, M. Rycenga, W. Li, and Y. Xia, "Measuring the SERS enhancement factors of dimers with different structures constructed from silver nanocubes," Chemical Physics Letters, vol. 484, no. 4-6, pp. 304-308, 2010.

[24] R. P. Van Duyne, J. C. Hulteen, and D. A. Treichel, "Atomic force microscopy and surface-enhanced Raman spectroscopy. I. Ag island films and Ag film over polymer nanosphere surfaces supported on glass," Journal of Chemical Physics, vol. 99, no. 3, pp. 2101-2115, 1993.

[25] X. Wang, W. Shi, G. She, and L. Mu, "Surface-Enhanced Raman Scattering (SERS) on transition metal and semiconductor nanostructures," Physical Chemistry Chemical Physics, vol. 14, no. 17, pp. 5891-5901, 2012.

[26] Y. B. Wu, Y. F. Wang, and X. W. Cao, "On the enhanced Raman scattering of the nanosize semiconductor: a couple of cylinders (silicon and silver)," Journal of Applied Physics, vol. 106, no. 5, Article ID 053106, 2009.

[27] A. K. Pradhan, R. B. Konda, H. Mustafa et al., "Surface plasmon resonance in CdSe semiconductor coated with gold nanoparticles," Optics Express, vol. 16, no. 9, pp. 6202-6208, 2008.

[28] K. C. See, J. B. Spicer, J. Brupbacher, D. Zhang, and T. G. Vargo, "Modeling interband transitions in silver nanoparticlefluoropolymer composites," Journal of Physical Chemistry B, vol. 109, no. 7, pp. 2693-2698, 2005.

[29] P. R. West, S. Ishii, G. V. Naik, N. K. Emani, V. M. Shalaev, and A. Boltasseva, "Searching for better plasmonic materials," Laser and Photonics Reviews, vol. 4, no. 6, pp. 795-808, 2010.

[30] E. Le Ru and P. Etchegoin, "Appendix E: dielectric function of gold and silver," in Principles of Surface-Enhanced Raman Spectroscopy: and Related Plasmonic Effects, pp. 529-535, Elsevier, New York, NY, USA, 2009.

[31] B. J. Kennedy, S. Spaeth, M. Dickey, and K. T. Carron, "Determination of the distance dependence and experimental effects for modified sers substrates based on self-assembled monolayers formed using alkanethiols," The Journal of Physical Chemistry B, vol. 103, no. 18, pp. 3640-3646, 1999.

[32] A. K. Singh, S. A. Khan, Z. Fan et al., "Development of a longrange surface-enhanced raman spectroscopy ruler," Journal of the American Chemical Society, vol. 134, no. 20, pp. 8662-8669, 2012.

[33] J. A. Dieringer, A. D. McFarland, N. C. Shah et al., "Surface enhanced Raman spectroscopy: new materials, concepts, characterization tools, and applications," Faraday Discussions, vol. 132, pp. 9-26, 2006.

[34] V. I. Kukushkin, A. B. Van'kov, and I. V. Kukushkin, "Longrange manifestation of surfaceenhanced Raman scattering," JETP Letters, vol. 98, no. 2, pp. 64-69, 2013.

[35] K. Kneipp, M. Moskovits, and H. Kneipp, Surface-Enhanced Raman Scattering: Physics and Applications, Springer Science \& Business Media, Berlin, Germany, 2006.
[36] A. D. McFarland, M. A. Young, J. A. Dieringer, and R. P. Van Duyne, "Wavelength-scanned surface-enhanced Raman excitation spectroscopy," The Journal of Physical Chemistry B, vol. 109, no. 22, pp. 11279-11285, 2005.

[37] N. Félidj, J. Aubard, G. Lévi et al., "Optimized surface-enhanced Raman scattering on gold nanoparticle arrays," Applied Physics Letters, vol. 82, no. 18, pp. 3095-3097, 2003.

[38] C. L. Haynes and R. P. Van Duyne, "Plasmon-sampled surfaceenhanced Raman excitation spectroscopy," Journal of Physical Chemistry B, vol. 107, no. 30, pp. 7426-7433, 2003.

[39] J. Grand, M. L. de la Chapelle, J.-L. Bijeon, P.-M. Adam, A. Vial, and P. Royer, "Role of localized surface plasmons in surfaceenhanced Raman scattering of shape-controlled metallic particles in regular arrays," Physical Review B: Condensed Matter and Materials Physics, vol. 72, no. 3, Article ID 033407, 2005.

[40] N. Guillot and M. L. de la Chapelle, "The electromagnetic effect in surface enhanced Raman scattering: enhancement optimization using precisely controlled nanostructures," Journal of Quantitative Spectroscopy and Radiative Transfer, vol. 113, no. 18, pp. 2321-2333, 2012.

[41] B. Sharma, R. R. Frontiera, A. I. Henry, E. Ringe, and R. P. Van Duyne, "SERS: materials, applications, and the future," Materials Today, vol. 15, no. 1-2, pp. 16-25, 2012.

[42] NanoComposix, “Tools," NanoComposix, 2014, http://nanocomposix.com/pages/tools.

[43] M. Quinten, Optical Properties of Nanoparticle Systems: Mie and Beyond, Wiley-VCH, Weinheim, Germany, 2011.

[44] E. Le Ru and P. Etchegoin, "Appendix H: Mie theory and its implementation," in Principles of Surface-Enhanced Raman Spectroscopy: and Related Plasmonic Effects, pp. 589-628, Elsevier, 2009.

[45] L. Qiao, D. Wang, L. Zuo et al., "Localized surface plasmon resonance enhanced organic solar cell with gold nanospheres," Applied Energy, vol. 88, no. 3, pp. 848-852, 2011.

[46] T. A. El-Brolossy, T. Abdallah, M. B. Mohamed et al., "Shape and size dependence of the surface plasmon resonance of gold nanoparticles studied by Photoacoustic technique," The European Physical Journal: Special Topics, vol. 153, no. 1, pp. 361364, 2008.

[47] E. Ringe, J. Zhang, M. R. Langille et al., "Effect of size, shape, composition, and support film on localized surface plasmon resonance frequency: a single particle approach applied to silver bipyramids and gold and silver nanocubes," in Proceedings of the Materials Research Society Fall Meeting, vol. 1208, 2010.

[48] S. Hong and X. Li, "Optimal size of gold nanoparticles for surface-enhanced Raman spectroscopy under different conditions," Journal of Nanomaterials, vol. 2013, Article ID 790323, 9 pages, 2013.

[49] V. Joseph, A. Matschulat, J. Polte, S. Rolf, F. Emmerling, and J. Kneipp, "SERS enhancement of gold nanospheres of defined size," Journal of Raman Spectroscopy, vol. 42, no. 9, pp. 17361742, 2011.

[50] M. Moskovits, "Surface-enhanced Raman spectroscopy: a brief retrospective," Journal of Raman Spectroscopy, vol. 36, no. 6-7, pp. 485-496, 2005.

[51] K. G. Stamplecoskie, J. C. Scaiano, V. S. Tiwari, and H. Anis, "Optimal size of silver nanoparticles for surface-enhanced raman spectroscopy," Journal of Physical Chemistry C, vol. 115, no. 5, pp. 1403-1409, 2011.

[52] P. N. Njoki, I.-I. S. Lim, D. Mott et al., "Size correlation of optical and spectroscopic properties for gold nanoparticles," The 
Journal of Physical Chemistry C, vol. 111, no. 40, pp. 14664-14669, 2007.

[53] S. Abalde-Cela, P. Aldeanueva-Potel, C. Mateo-Mateo, L. Rodríguez-Lorenzo, R. A. Alvarez-Puebla, and L. M. LizMarzán, "Surface-enhanced Raman scattering biomedical applications of plasmonic colloidal particles," Journal of the Royal Society Interface, vol. 7, no. 4, pp. S435-S450, 2010.

[54] H.-Y. Wu, W.-L. Huang, and M. H. Huang, "Direct high-yield synthesis of high aspect ratio gold nanorods," Crystal Growth and Design, vol. 7, no. 4, pp. 831-835, 2007.

[55] C. B. Moore, W. Rison, J. Mathis, and G. Aulich, "Lightning rod improvement studies," Journal of Applied Meteorology, vol. 39, no. 5, pp. 593-609, 2000.

[56] J. Lee, B. Hua, S. Park et al., "Tailoring surface plasmons of highdensity gold nanostar assemblies on metal films for surfaceenhanced Raman spectroscopy," Nanoscale, vol. 6, no. 1, pp. 616623, 2014.

[57] U. Gaware, V. Kamble, and B. Ankamwar, "Ecofriendly synthesis of anisotropic gold nanoparticles: a potential candidate of SERS studies," International Journal of Electrochemistry, vol. 2012, Article ID e276246, 2012.

[58] P. Halevi, "Chapter 2: surface enhanced Raman scattering," in Photonic Probes of Surfaces, chapter 2, pp. 65-95, Newnes, New South Wales, Australia, 2012.

[59] J. Li, D. Fattal, and Z. Li, "Plasmonic optical antennas on dielectric gratings with high field enhancement for surface enhanced Raman spectroscopy," Applied Physics Letters, vol. 94, no. 26, Article ID 263114, 2009.

[60] M. Li, Z. S. Zhang, X. Zhang, K. Y. Li, and X. F. Yu, "Optical properties of $\mathrm{Au} / \mathrm{Ag}$ core/shell nanoshuttles," Optics Express, vol. 16, no. 18, pp. 14288-14293, 2008.

[61] E. C. Le Ru, J. Grand, I. Sow et al., "A scheme for detecting every single target molecule with surface-enhanced raman spectroscopy," Nano Letters, vol. 11, no. 11, pp. 5013-5019, 2011.

[62] A. Ermushev, B. Mchedlishvili, V. Oleinikov, and A. Petukhov, "Surface enhancement of local optical fields and the lightningrod effect," Kvantovaya Elektron, vol. 20, no. 5, pp. 503-508, 1993.

[63] V. G. Pol, H. Grisaru, and A. Gedanken, "Coating noble metal nanocrystals ( $\mathrm{Ag}, \mathrm{Au}, \mathrm{Pd}$, and $\mathrm{Pt}$ ) on polystyrene spheres via ultrasound irradiation," Langmuir, vol. 21, no. 8, pp. 3635-3640, 2005.

[64] A. Dokoutchaev, J. Thomas James, S. C. Koene, S. Pathak, G. K. Surya Prakash, and M. E. Thompson, "Colloidal metal deposition onto functionalized polystyrene microspheres," Chemistry of Materials, vol. 11, no. 9, pp. 2389-2399, 1999.

[65] Y. Kobayashi, Y. Tadaki, D. Nagao, and M. Konno, "Deposition of gold nanoparticles on polystyrene spheres by electroless metal plating technique," Journal of Physics: Conference Series, vol. 61, no. 1, pp. 582-586, 2007.

[66] Z. Deng, M. Chen, and L. Wu, "Novel method to fabricate $\mathrm{SiO}_{2} / \mathrm{Ag}$ composite spheres and their catalytic, surfaceenhanced raman scattering properties," The Journal of Physical Chemistry C, vol. 111, no. 31, pp. 11692-11698, 2007.

[67] J. H. Kim, W. W. Bryan, and R. T. Lee, "Preparation, characterization, and optical properties of gold, silver, and gold-silver alloy nanoshells having silica cores," Langmuir, vol. 24, no. 19, pp. 11147-11152, 2008.

[68] Y. T. Lim, O. O. Park, and H.-T. Jung, "Gold nanolayerencapsulated silica particles synthesized by surface seeding and shell growing method: near infrared responsive materials,"
Journal of Colloid and Interface Science, vol. 263, no. 2, pp. 449453, 2003.

[69] I. Y. Goon, L. M. H. Lai, M. Lim, P. Munroe, J. J. Gooding, and R. Amal, "Fabrication and dispersion of gold-shellprotected magnetite nanoparticles: systematic control using polyethyleneimine," Chemistry of Materials, vol. 21, no. 4, pp. 673-681, 2009.

[70] L. Zhang, J. Xu, L. Mi, H. Gong, S. Jiang, and Q. Yu, "Multifunctional magnetic-plasmonic nanoparticles for fast concentration and sensitive detection of bacteria using SERS," Biosensors and Bioelectronics, vol. 31, no. 1, pp. 130-136, 2012.

[71] V. K. Pustovalov and W. Fritzsche, "Nonlinear dependences of optical properties of spherical core-shell silver-gold and goldsilver nanoparticles on their parameters," Plasmonics, vol. 8, no. 2, pp. 983-993, 2013.

[72] S. Pande, S. K. Ghosh, S. Praharaj et al., "Synthesis of normal and inverted gold-silver core-shell architectures in $\beta$-cyclodextrin and their applications in SERS," The Journal of Physical Chemistry C, vol. 111, no. 29, pp. 10806-10813, 2007.

[73] Z. Y. Li, J. P. Wilcoxon, F. Yin, Y. Chen, R. E. Palmer, and R. L. Johnston, "Structures and optical properties of 4-5 nm bimetallic AgAu nanoparticles," Faraday Discussions, vol. 138, pp. 363-373, 2008.

[74] P. K. Jain, K. S. Lee, I. H. El-Sayed, and M. A. El-Sayed, "Calculated absorption and scattering properties of gold nanoparticles of different size, shape, and composition: applications in biological imaging and biomedicine," Journal of Physical Chemistry B, vol. 110, no. 14, pp. 7238-7248, 2006.

[75] S. J. Oldenburg, R. D. Averitt, S. L. Westcott, and N. J. Halas, "Nanoengineering of optical resonances," Chemical Physics Letters, vol. 288, no. 2-4, pp. 243-247, 1998.

[76] Y. Ma, J. Zhou, W. Zou, Z. Jia, L. Petti, and P. Mormile, "Localized surface plasmon resonance and surface enhanced raman scattering responses of Au@Ag core-shell nanorods with different thickness of Ag shell," Journal of Nanoscience and Nanotechnology, vol. 14, no. 6, pp. 4245-4250, 2014.

[77] L. Lu, H. Wang, Y. Zhou et al., "Seed-mediated growth of large, monodisperse core-shell gold-silver nanoparticles with Ag-like optical properties," Chemical Communications, no. 2, pp. 144$145,2002$.

[78] E. Csapo, A. Oszko, E. Varga et al., "Synthesis and characterization of Ag/Au alloy and core(Ag)-shell( $\mathrm{Au})$ nanoparticles," Colloids and Surfaces A, Physicochemical and Engineering Aspects, vol. 415, pp. 281-287, 2012.

[79] D. Mott, J. Lee, N. T. B. Thuy, Y. Aoki, P. Singh, and S. Maenosono, "A study on the plasmonic properties of silver core gold shell nanoparticles: optical assessment of the particle structure," Japanese Journal of Applied Physics, vol. 50, no. 6, Article ID 065004, 2011.

[80] L. Lu, G. Burkey, I. Halaciuga, and D. V. Goia, "Core-shell gold/silver nanoparticles: synthesis and optical properties," Journal of Colloid and Interface Science, vol. 392, no. 1, pp. 90-95, 2013.

[81] H. Liu, L. Zhang, X. Lang et al., "Single molecule detection from a large-scale SERS-active Au 79Ag21 substrate," Scientific Reports, vol. 1, article 112, 2011.

[82] S. Nie and S. R. Emory, "Probing single molecules and single nanoparticles by surface-enhanced Raman scattering," Science, vol. 275, no. 5303, pp. 1102-1106, 1997.

[83] H. M. Lee, S. M. Jin, H. M. Kim, and Y. D. Suh, "Single-molecule surface-enhanced Raman spectroscopy: a perspective on the 
current status," Physical Chemistry Chemical Physics, vol. 15, no. 15, pp. 5276-5287, 2013.

[84] D.-K. Lim, K.-S. Jeon, H. M. Kim, J.-M. Nam, and Y. D. Suh, "Nanogap-engineerable raman-active nanodumbbells for single-molecule detection," Nature Materials, vol. 9, no. 1, pp. 60-67, 2010.

[85] K. Faulds, F. McKenzie, W. E. Smith, and D. Graham, "Quantitative simultaneous multianalyte detection of DNA by dualwavelength surface-enhanced resonance raman scattering," Angewandte Chemie International Edition, vol. 46, no. 11, pp. 1829-1831, 2007.

[86] A. Kudelski, "Raman studies of rhodamine 6G and crystal violet sub-monolayers on electrochemically roughened silver substrates: do dye molecules adsorb preferentially on highly SERS-active sites?" Chemical Physics Letters, vol. 414, no. 4-6, pp. 271-275, 2005.

[87] P. Hildebrandt and M. Stockhurger, "Surface-enhanced resonance Raman spectroscopy of rhodamine $6 \mathrm{G}$ adsorbed on colloidal silver," Journal of Physical Chemistry, vol. 88, no. 24, pp. 5935-5944, 1984.

[88] J. A. Dieringer, K. L. Wustholz, D. J. Masiello et al., "Surfaceenhanced Raman excitation spectroscopy of a single rhodamine $6 \mathrm{G}$ molecule," Journal of the American Chemical Society, vol. 131, no. 2, pp. 849-854, 2009.

[89] N. P. W. Pieczonka, G. Moula, and R. F. Aroca, "SERRS for single-molecule detection of dye-labeled phospholipids in Langmuir-Blodgett monolayers," Langmuir, vol. 25, no. 19, pp. 11261-11264, 2009.

[90] M. V. Cañamares, C. Chenal, R. L. Birke, and J. R. Lombardi, "DFT, SERS, and single-molecule SERS of crystal violet," Journal of Physical Chemistry C, vol. 112, no. 51, pp. 20295-20300, 2008.

[91] X. Qian, S. R. Emory, and S. Nie, "Anchoring molecular chromophores to colloidal gold nanocrystals: surface-enhanced Raman evidence for strong electronic coupling and irreversible structural locking," Journal of the American Chemical Society, vol. 134, no. 4, pp. 2000-2003, 2012.

[92] A. M. Fales, H. Yuan, and T. Vo-Dinh, "Silica-coated gold nanostars for combined surface-enhanced Raman scattering (SERS) detection and singlet-oxygen generation: a potential nanoplatform for theranostics," Langmuir, vol. 27, no. 19, pp. 12186-12190, 2011.

[93] S. H. A. Nicolai and J. C. Rubim, "Surface-enhanced resonance Raman (SERR) spectra of methylene blue adsorbed on a silver electrode," Langmuir, vol. 19, no. 10, pp. 4291-4294, 2003.

[94] H.-X. Cai, Y. Zhang, K.-T. Yong, Z. Shao, Y.-X. Li, and X.-H. Zhang, "Raman spectrum study of $3.3^{\prime}$-diethylthiatri carbocyanine iodide," Spectroscopy and Spectral Analysis, vol. 30, no. 12, pp. 3244-3248, 2010.

[95] E. Smith and G. Dent, "Chapter 4: resonance Raman scattering," in Modern Raman Spectroscopy-A Practical Approach, pp. 93112, John Wiley \& Sons, New York, NY, USA, 2005.

[96] D. A. Long, "Intensities in Raman spectra. I. A bond polarizability theory," Proceedings of the Royal Society A: Mathematical, Physical and Engineering Sciences, vol. 217, no. 1129, pp. 203-221, 1953.

[97] P. G. Etchegoin, P. D. Lacharmoise, and E. C. Le Ru, "Influence of photostability on single-molecule surface enhanced Raman scattering enhancement factors," Analytical Chemistry, vol. 81, no. 2, pp. 682-688, 2009.

[98] Y. Fang, N.-H. Seong, and D. D. Dlott, "Measurement of the distribution of site enhancements in surface-enhanced raman scattering," Science, vol. 321, no. 5887, pp. 388-392, 2008.
[99] N. G. Greeneltch, A. S. Davis, N. A. Valley et al., "Nearinfrared surface-enhanced raman spectroscopy (NIR-SERS) for the identification of eosin Y: theoretical calculations and evaluation of two different nanoplasmonic substrates," The Journal of Physical Chemistry A, vol. 116, no. 48, pp. 11863-11869, 2012.

[100] P. Šimáková, J. Gautier, M. Procházka, K. Hervé-Aubert, and I. Chourpa, "Polyethylene-glycol-stabilized ag nanoparticles for surface-enhanced raman scattering spectroscopy: Ag surface accessibility studied using metalation of free-base porphyrins," Journal of Physical Chemistry C, vol. 118, no. 14, pp. 7690-7697, 2014.

[101] W. E. Doering and S. M. Nie, "Spectroscopic tags using dyeembedded nanoparticles and surface-enhanced Raman scattering," Analytical Chemistry, vol. 75, no. 22, pp. 6171-6176, 2003.

[102] X. Su, J. Zhang, L. Sun et al., "Composite Organic-Inorganic Nanoparticles (COINs) with chemically encoded optical signatures," Nano Letters, vol. 5, no. 1, pp. 49-54, 2005.

[103] C.-C. Huang, C.-H. Huang, I.-T. Kuo, L.-K. Chau, and T.-S. Yang, "Synthesis of silica-coated gold nanorod as Raman tags by modulating cetyltrimethylammonium bromide concentration," Colloids and Surfaces A: Physicochemical and Engineering Aspects, vol. 409, pp. 61-68, 2012.

[104] L. Rocks, K. Faulds, and D. Graham, "Rationally designed SERS active silica coated silver nanoparticles," Chemical Communications, vol. 47, no. 15, pp. 4415-4417, 2011.

[105] G. Schmid, "Large clusters and colloids. Metals in the embryonic state," Chemical Reviews, vol. 92, no. 8, pp. 1709-1727, 1992.

[106] J. Turkevich, P. C. Stevenson, and J. Hillier, "A study of the nucleation and growth processes in the synthesis of colloidal gold," Discussions of the Faraday Society, vol. 11, pp. 55-75, 1951.

[107] M. Brust, M. Walker, D. Bethell, D. J. Schiffrin, and R. Whyman, "Synthesis of thiol-derivatised gold nanoparticles in a twophase liquid-liquid system," Journal of the Chemical Society, Chemical Communications, no. 7, pp. 801-802, 1994.

[108] P. C. Lee and D. Meisel, "Adsorption and surface-enhanced Raman of dyes on silver and gold sols," Journal of Physical Chemistry, vol. 86, no. 17, pp. 3391-3395, 1982.

[109] J. A. Creighton, C. G. Blatchford, and M. G. Albrecht, "Plasma resonance enhancement of Raman scattering by pyridine adsorbed on silver or gold sol particles of size comparable to the excitation wavelength," Journal of the Chemical Society, Faraday Transactions 2: Molecular and Chemical Physics, vol. 75, pp. 790798, 1979.

[110] R. A. Caruso, M. Ashokkumar, and F. Grieser, "Sonochemical formation of gold sols," Langmuir, vol. 18, no. 21, pp. 7831-7836, 2002.

[111] K. Okitsu, K. Sharyo, and R. Nishimura, "One-pot synthesis of gold nanorods by ultrasonic irradiation: the effect of $\mathrm{pH}$ on the shape of the gold nanorods and nanoparticles," Langmuir, vol. 25, no. 14, pp. 7786-7790, 2009.

[112] Y. Liu, L. He, C. Xu, and M. Han, "Photochemical fabrication of hierarchical Ag nanoparticle arrays from domain-selective $\mathrm{Ag}^{+}$loading on block copolymer templates," Chemical Communications, no. 43, pp. 6566-6568, 2009.

[113] L. M. Alrehaily, J. M. Joseph, M. C. Biesinger, D. A. Guzonas, and J. C. Wren, "Gamma-radiolysis-assisted cobalt oxide nanoparticle formation," Physical Chemistry Chemical Physics, vol. 15, no. 3, pp. 1014-1024, 2013.

[114] D. Zopes, B. Stein, S. Mathur, and C. Graf, "Improved stability of "naked" gold nanoparticles enabled by in situ coating with 
mono and multivalent thiol PEG ligands," Langmuir, vol. 29, no. 36, pp. 11217-11226, 2013.

[115] T. K. Sau and C. J. Murphy, "Seeded high yield synthesis of short Au nanorods in aqueous solution," Langmuir, vol. 20, no. 15, pp. 6414-6420, 2004.

[116] S.-Y. Zhao, S.-H. Chen, D.-G. Li, X.-G. Yang, and H.-Y. Ma, "A convenient phase transfer route for Ag nanoparticles," Physica E: Low-Dimensional Systems and Nanostructures, vol. 23, no. 12, pp. 92-96, 2004

[117] N. Leopold and B. Lendl, "A new method for fast preparation of highly surface-enhanced raman scattering (SERS) active silver colloids at room temperature by reduction of silver nitrate with hydroxylamine hydrochloride," The Journal of Physical Chemistry B, vol. 107, no. 24, pp. 5723-5727, 2003.

[118] S. Kittler, C. Greulich, M. Köller, and M. Epple, "Synthesis of PVP-coated silver nanoparticles and their biological activity towards human mesenchymal stem cells," Materialwissenschaft und Werkstofftechnik, vol. 40, no. 4, pp. 258-264, 2009.

[119] E. A. Hauser and J. E. Lynn, Experiments in Colloid Chemistry, McGraw-Hill, 1940.

[120] Y. Wan, Z. Guo, X. Jiang et al., "Quasi-spherical silver nanoparticles: aqueous synthesis and size control by the seed-mediated Lee-Meisel method," Journal of Colloid and Interface Science, vol. 394, no. 1, pp. 263-268, 2013.

[121] J. Kimling, M. Maier, B. Okenve, V. Kotaidis, H. Ballot, and A. Plech, "Turkevich method for gold nanoparticle synthesis revisited," Journal of Physical Chemistry B, vol. 110, no. 32, pp. 15700-15707, 2006.

[122] E. A. Vitol, G. Friedman, and Y. Gogotsi, "Surface-enhanced raman spectroscopy-active substrates: adapting the shape of plasmonic nanoparticles for different biological applications," Journal of Nanoscience and Nanotechnology, vol. 14, no. 4, pp. 3046-3051, 2014.

[123] X. Dong, X. Ji, H. Wu, L. Zhao, J. Li, and W. Yang, "Shape control of silver nanoparticles by stepwise citrate reduction," The Journal of Physical Chemistry C, vol. 113, no. 16, pp. 65736576, 2009.

[124] G. T. Hermanson, "Preparation of colloidal gold-labeled proteins," in Bioconjugate Techniques, chapter 24, pp. 924-935, Elsevier, New York, NY, USA, 2nd edition, 2008.

[125] W. Niu, L. Zhang, and G. Xu, "Seed-mediated growth of noble metal nanocrystals: crystal growth and shape control," Nanoscale, vol. 5, no. 8, pp. 3172-3181, 2013.

[126] A. K. Samal, T. S. Sreeprasad, and T. Pradeep, "Investigation of the role of $\mathrm{NaBH} 4$ in the chemical synthesis of gold nanorods," Journal of Nanoparticle Research, vol. 12, no. 5, pp. 1777-1786, 2010.

[127] N. R. Jana, L. Gearheart, and C. J. Murphy, "Evidence for seedmediated nucleation in the chemical reduction of gold salts to gold nanoparticles," Chemistry of Materials, vol. 13, no. 7, pp. 2313-2322, 2001.

[128] N. R. Jana, L. Gearheart, and C. J. Murphy, "Seeding growth for size control of 5-40 nm diameter gold nanoparticles," Langmuir, vol. 17, no. 22, pp. 6782-6786, 2001.

[129] S. Kumar, H. Yang, and S. Zou, "Seed-mediated growth of uniform gold nanoparticle arrays," Journal of Physical Chemistry C, vol. 111, no. 35, pp. 12933-12938, 2007.

[130] H. You, S. Yang, B. Ding, and H. Yang, "Synthesis of colloidal metal and metal alloy nanoparticles for electrochemical energy applications," Chemical Society Reviews, vol. 42, no. 7, pp. 28802904, 2013.
[131] C. J. Orendorff, L. Gearheart, N. R. Jana, and C. J. Murphy, "Aspect ratio dependence on surface enhanced Raman scattering using silver and gold nanorod substrates," Physical Chemistry Chemical Physics, vol. 8, no. 1, pp. 165-170, 2006.

[132] S. Y. Lee, L. Hung, G. S. Lang, J. E. Cornett, I. D. Mayergoyz, and O. Rabin, "Dispersion in the SERS enhancement with silver nanocube dimers," ACS Nano, vol. 4, no. 10, pp. 5763-5772, 2010.

[133] C. G. Khoury and T. Vo-Dinh, "Gold nanostars for surfaceenhanced Raman scattering: synthesis, characterization and optimization," The Journal of Physical Chemistry C, vol. 112, no. 48, pp. 18849-18859, 2008.

[134] D. Correia-Ledo, K. F. Gibson, A. Dhawan et al., "Assessing the location of surface plasmons over nanotriangle and nanohole arrays of different size and periodicity," Journal of Physical Chemistry C, vol. 116, no. 12, pp. 6884-6892, 2012.

[135] T. Tan, C. Tian, Z. Ren et al., "LSPR-dependent SERS performance of silver nanoplates with highly stable and broad tunable LSPRs prepared through an improved seed-mediated strategy," Physical Chemistry Chemical Physics, vol. 15, no. 48, pp. 2103421042, 2013.

[136] H. Wang, X. Han, X. Ou, C.-S. Lee, X. Zhang, and S.-T. Lee, "Silicon nanowire based single-molecule SERS sensor," Nanoscale, vol. 5, no. 17, pp. 8172-8176, 2013.

[137] L. R. Bickford, G. Agollah, R. Drezek, and T.-K. Yu, "Silicagold nanoshells as potential intraoperative molecular probes for HER2-overexpression in ex vivo breast tissue using near-infrared reflectance confocal microscopy," Breast Cancer Research and Treatment, vol. 120, no. 3, pp. 547-555, 2010.

[138] L. R. Hirsch, J. B. Jackson, A. Lee, N. J. Halas, and J. L. West, "A whole blood immunoassay using gold nanoshells," Analytical Chemistry, vol. 75, no. 10, pp. 2377-2381, 2003.

[139] J. Song, B. Duan, C. Wang et al., "SERS-encoded nanogapped plasmonic nanoparticles: growth of metallic nanoshell by templating redox-active polymer brushes," Journal of the American Chemical Society, vol. 136, no. 19, pp. 6838-6841, 2014.

[140] G. H. Woehrle, L. O. Brown, and J. E. Hutchison, "Thiolfunctionalized, 1.5-nm gold nanoparticles through ligand exchange reactions: scope and mechanism of ligand exchange," Journal of the American Chemical Society, vol. 127, no. 7, pp. 2172-2183, 2005.

[141] R. Stiufiuc, C. Iacovita, R. Nicoara et al., "One-step synthesis of PEGylated gold nanoparticles with tunable surface charge," Journal of Nanomaterials, vol. 2013, Article ID 146031, 7 pages, 2013.

[142] S. P. Mulvaney, M. D. Musick, C. D. Keating, and M. J. Natan, "Glass-coated, analyte-tagged nanoparticles: a new tagging system based on detection with surface-enhanced Raman scattering," Langmuir, vol. 19, no. 11, pp. 4784-4790, 2003.

[143] J.-M. Li, C. Wei, W.-F. Ma et al., "Multiplexed SERS detection of DNA targets in a sandwich-hybridization assay using SERSencoded core-shell nanospheres," Journal of Materials Chemistry, vol. 22, no. 24, pp. 12100-12106, 2012.

[144] C. M. Cobley, L. Au, J. Chen, and Y. Xia, “Targeting gold nanocages to cancer cells for photothermal destruction and drug delivery," Expert Opinion on Drug Delivery, vol. 7, no. 5, pp. 577-587, 2010.

[145] A. Weiss, T. C. Preston, J. Popov et al., "Selective recognition of rituximab-functionalized gold nanoparticles by lymphoma cells studied with 3D imaging," Journal of Physical Chemistry C, vol. 113, no. 47, pp. 20252-20258, 2009.

[146] G. T. Hermanson, Bioconjugate Techniques, Academic Press, 2010. 
[147] L. Levy, Y. Sahoo, K.-S. Kim, E. J. Bergey, and P. N. Prasad, "Nanochemistry: synthesis and characterization of multifunctional nanoclinics for biological applications," Chemistry of Materials, vol. 14, no. 9, pp. 3715-3721, 2002.

[148] J. Kneipp, H. Kneipp, A. Rajadurai, R. W. Redmond, and K. Kneipp, "Optical probing and imaging of live cells using SERS labels," Journal of Raman Spectroscopy, vol. 40, no. 1, pp. 1-5, 2009.

[149] A. Hornemann, D. Drescher, S. Flemig, and J. Kneipp, "Intracellular SERS hybrid probes using BSA-reporter conjugates," Analytical and Bioanalytical Chemistry, vol. 405, no. 19, pp. 6209-6222, 2013.

[150] G. L. Liu, F. F. Chen, J. A. Ellman, and L. P. Lee, "Peptidenanoparticle hybrid SERS probe for dynamic detection of active cancer biomarker enzymes," Conference Proceedings: Annual International Conference of the IEEE Engineering in Medicine and Biology Society, vol. 1, pp. 795-798, 2006.

[151] M. D. Hodges, J. G. Kelly, A. J. Bentley et al., "Combining immunolabeling and surface-enhanced Raman spectroscopy on cell membranes," ACS Nano, vol. 5, no. 12, pp. 9535-9541, 2011.

[152] Y. Wang, L.-J. Tang, and J.-H. Jiang, "Surface-enhanced raman spectroscopy-based, homogeneous, multiplexed immunoassay with antibody-fragments-decorated gold nanoparticles," Analytical Chemistry, vol. 85, no. 19, pp. 9213-9220, 2013. 

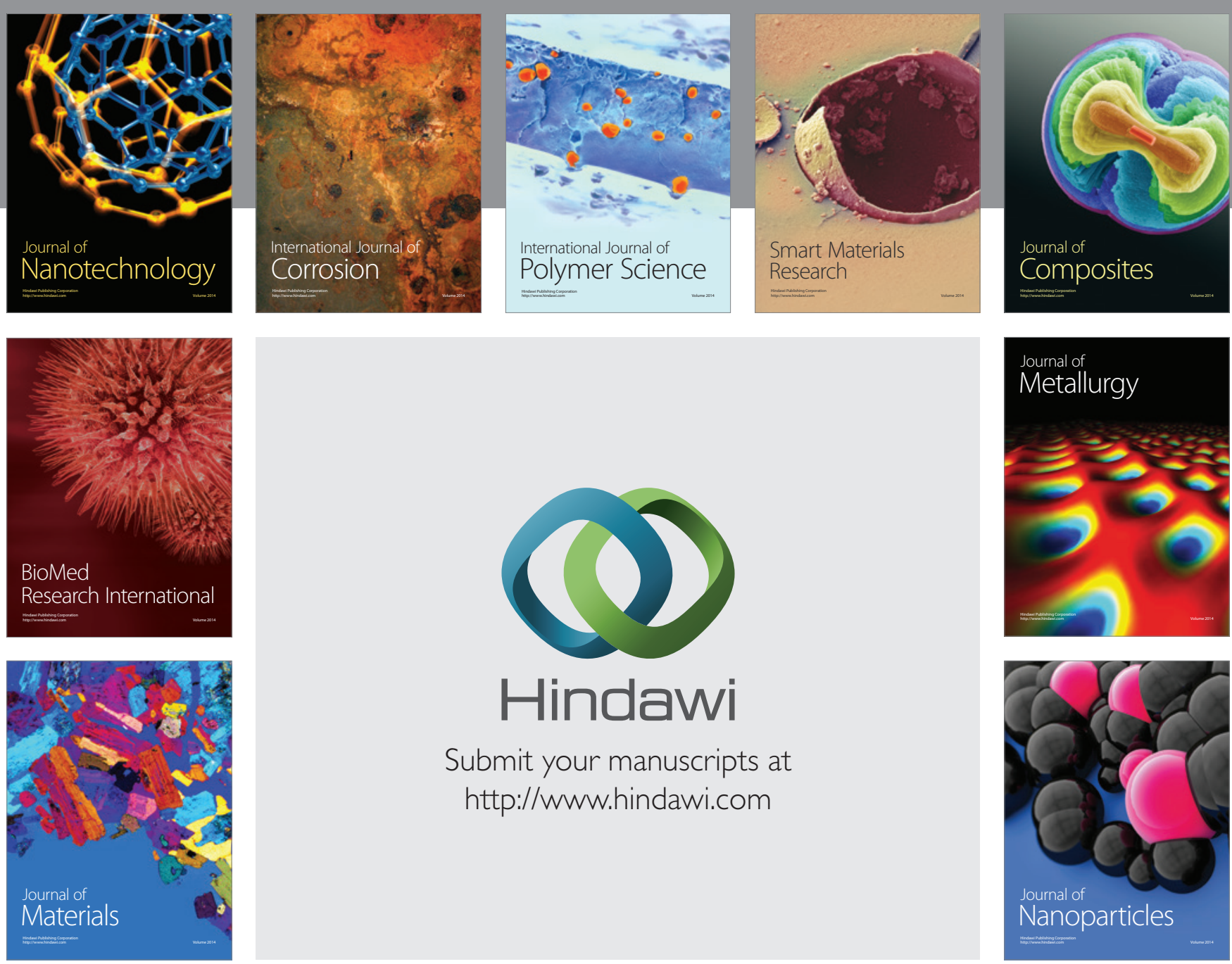

Submit your manuscripts at http://www.hindawi.com
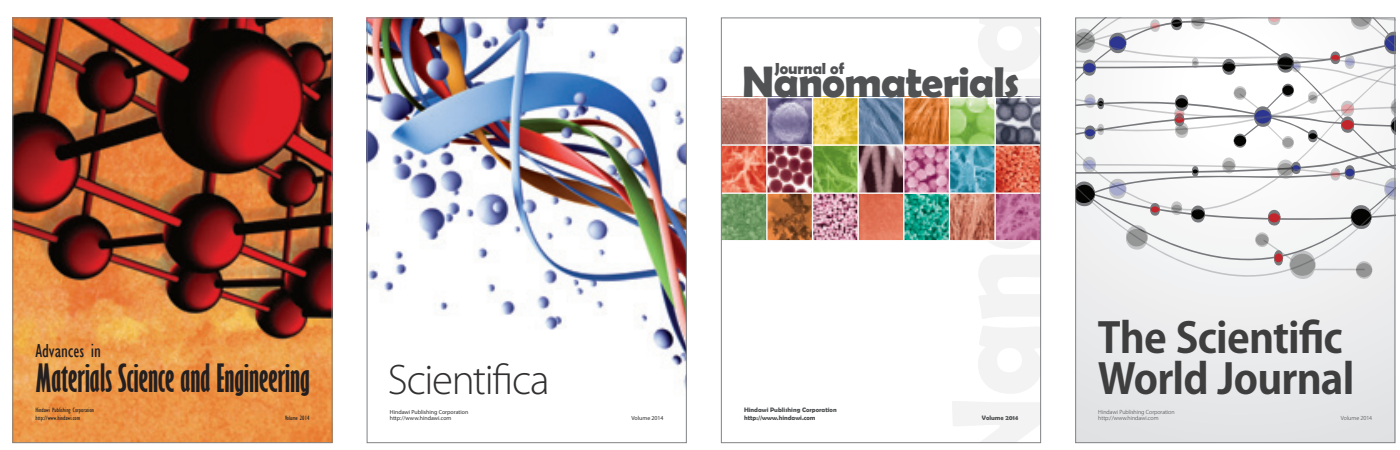

\section{The Scientific World Journal}
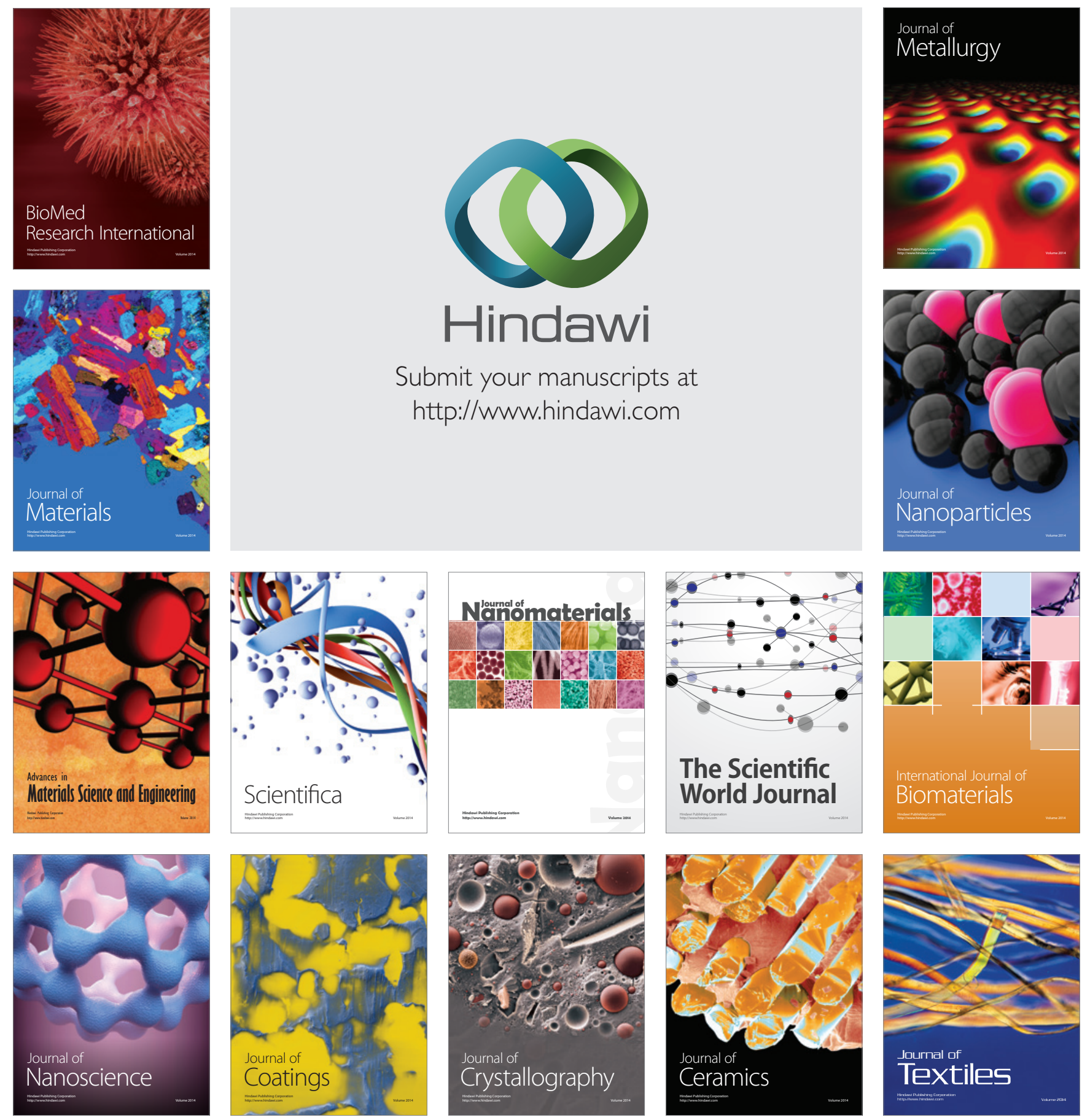